\title{
Respiratory infection and primary immune deficiency - what does the general physician need to know?
}

\author{
${ }^{1} \mathrm{~S}$ Lear, ${ }^{2} \mathrm{~A}$ Condliffe \\ ${ }^{1}$ Registrar in Clinical Immunology, Department of Clinical Immunology; ${ }^{2}$ University Lecturer and Honorary Consultant in Respiratory Medicine, \\ Department of Medicine, Addenbrooke's Hospital, Cambridge, UK
}

\begin{abstract}
This review, based on a talk given at the RCPE Respiratory Medicine Symposium 2014, outlines the clinical spectrum of immune deficiency - antibody (B cell) deficiency, $T$ cell defects and innate/phagocytic disorders - and discusses the relevant clinical presentations, investigations and treatments, focusing particularly on the management of adults with recurrent respiratory infections. It describes when to suspect a primary immunodeficiency, the first-line investigations to perform and the triggers that should prompt referral for further specialist opinion. The paper concludes with a look at a novel primary immunodeficiency, Activated PI3 Kinase Delta Syndrome (APDS).
\end{abstract}

KEYWORDS Activated PI3 Kinase Delta Syndrome (APDS), antibody deficiency, common variable immunodeficiency, immunoglobulin, immunological screening, primary immunodeficiency

DECLARATIONS OF INTERESTS Dr Condliffe has received non-commercial research grants to support laboratory studies (not supporting the work in the article but for other research) from GSK and Medlmmune and has undertaken consultancy work for GSK regarding PI3 kinase signalling.

\section{INTRODUCTION}

Primary immunodeficiency (PID) encompasses a group of inherited (monogeneic or polygeneic) conditions in which a measurable decrease in the quantity or function of one or more immune components (Figure I) is associated with clinical disease. About 5,000 individuals in the UK are currently estimated to suffer from a PID, although the nature and severity is highly variable; to obtain more detailed information the UK-PID Registry has been established, and to date more than 2200 patients have been enrolled in this ongoing initiative.' In general, the more severely impaired the immune system, the earlier the presentation. Increasingly, milder PIDs are being diagnosed in the adult population. ${ }^{2,3}$ This reflects an increased awareness of conditions such as antibody deficiency, plus better access to diagnostic tests and specialist services, leading to an improving diagnostic (and hence treatment) rate.

Despite this increased awareness, there is still often a substantial diagnostic delay in some conditions; for example, patients diagnosed with common variable immunodeficiency (CVID) have symptoms of this disease for an average of five to seven years prior to diagnosis. ${ }^{4}$ These delays increase the risk that the patient will have irreversible end-organ damage before treatment is initiated. Most patients are identified on the basis of
Correspondence to A Condliffe University of Cambridge Department of Medicine Addenbrooke's Hospital Hills Road

Cambridge CB2 2QQ, UK

e-mail amc46@medschl.cam.ac.uk
INNATE

ADAPTIVE

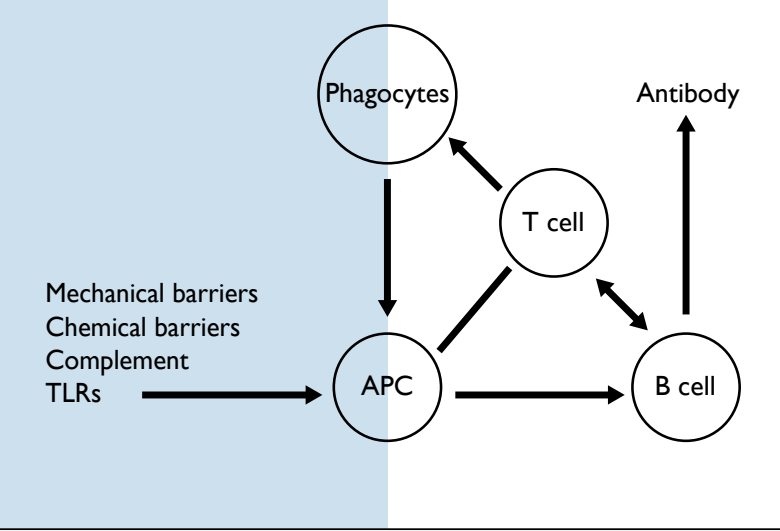

FIGURE I Compartments of the immune system. The innate immune system provides the first line of defense, mounting an immediate response to pathogen-associated molecules resulting in pathogen destruction and clearance. Antigen presenting cells (APC) activate lymphocytes during antigen presentation, allowing the adaptive immune system to generate pathogen-specific receptors on $\mathrm{T}$ and $\mathrm{B}$ lymphocytes; these lymphocytes then co-ordinate pathogen destruction (via activated CD8 cells and phagocytes) and establish a memory pool for future infection. Antibodies form a bridge between the systems - opsonisation targets pathogens for destruction by innate immune cells; furthermore, phagocytes are activated by cytokines and cellcell interaction with mainly CD4 cells. 
abnormal results detected in simple tests that can be performed in most NHS laboratories.

It is important that physicians in both primary and secondary care are aware that a) primary (as well as acquired) immune deficiencies may present in adulthood; b) a basic immunological screen will be readily available; c) a regional clinical immunology service is accessible to help, advise and facilitate investigation; and d) early referrals to immunology services are encouraged for patients with abnormal relevant test results or serious, persistent, unusual or highly recurrent infections. Recognition, appropriate investigation and referral are critical for these patients, because a specific and highly efficacious treatment is available for antibody deficiency states (immunoglobulin replacement) and because patients with many different PIDs may benefit from targeted anti-bacterial, anti-viral and anti-fungal prophylaxis. $^{5}$ These strategies can have a dramatic impact on life quality and improve life expectancy.

This review aims to outline in brief the clinical spectrum of immune deficiency - namely antibody (B cell) deficiency, $T$ cell defects and innate/phagocytic disorders - and to discuss the relevant clinical presentations (Table I), investigations and treatments (Figure 2). We focus particularly on the management of adults with recurrent respiratory infections. Very rare disorders or those in which respiratory disease is not a recognised feature are beyond the scope of this article. We will discuss when to suspect a PID, the first-line investigations to perform when PID is being considered, and the triggers that should prompt referral for further specialist opinion and investigation. Finally, we give an example of how nextgeneration sequencing technology may transform our understanding of PIDs and lead to the identification and characterisation of a novel PID with specific therapeutic options likely to be available in the near future.

\section{PRIMARY ANTIBODY DEFICIENCY (PAD)}

\section{When to suspect PAD}

Patients with a quantitative or qualitative immunoglobulin (Ig) defect can present at any age, most commonly with recurrent bacterial infections of the respiratory tract. Haemophilus influenzae and/or Streptococcus pneumoniae are the most commonly isolated pathogens; other organisms such as Pseudomonas aeruginosa may supervene if there is structural lung damage (such as bronchiectasis). ${ }^{6}$ Otitis media is common in children, and sinus disease is a feature in most patients.

The clinical picture is one of recurrent moderate episodes of infection requiring increasingly frequent courses of oral antibiotics, sickness absence from school or work, and progressive deconditioning. Patients with a lengthy history of infections may accept this as 'normal'

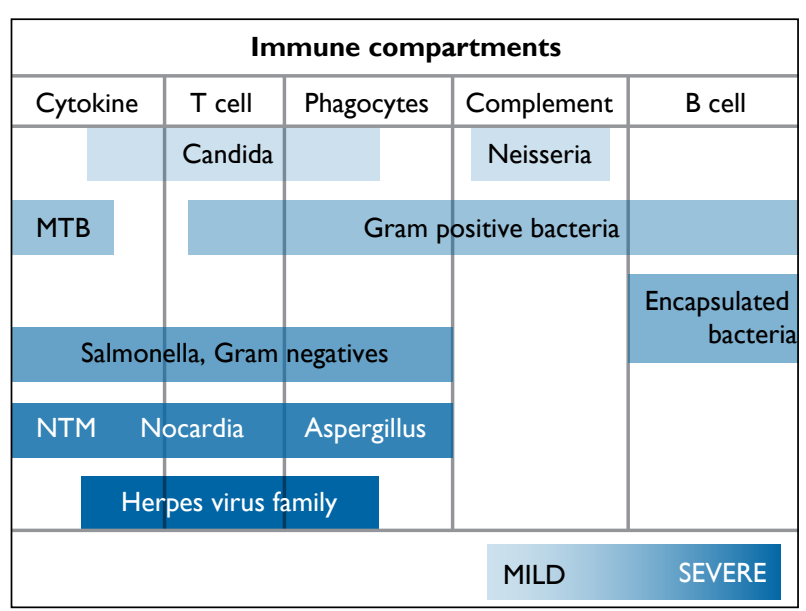

FIGURE 2 Pathogens associated with known immunodeficiency states. Dysfunction of immune system components leads to a characteristic spectrum of infections that may help in pattern recognition and flag up the possibility of an underlying PID.

for them, and such acceptance may prolong diagnostic delay. This pattern of recurrent mild infection may be punctuated by episodes of more severe invasive infection - pneumonia, septicaemia, osteomyelitis or meningitis, requiring hospital admission. Diarrhoea and/or malabsorption are seen and may be associated with persistent infection with gut pathogens, including giardia, salmonella and norovirus. If unrecognised, recurrent infection results in irreversible end-organ damage (in particular, hearing impairment and bronchiectasis) with associated morbidity and mortality.

Importantly, 20\% of patients with CVID develop autoimmunity, particularly autoimmune thrombocytopaenia or haemolytic anaemia, and these features may precede, coincide with or follow the onset of infection. More recently, it has been recognised that $10 \%$ of CVID patients also develop an aggressive form of interstitial lung disease, termed granulomatous lymphocytic interstitial lung disease (GLILD), and a similar proportion will suffer from $B$ cell malignancy. Hence recurrent severe oto-sinopulmonary infection, and in particular the co-existence of such infections with autoimmunity, interstitial lung disease or lymphoma, should prompt the question: 'Does this patient have an underlying PAD?'

\section{How to diagnose PAD}

This group includes any patient found to have reduced levels of total $\lg$, IgA or lgM, lgG subclasses or specific antibodies (functional vaccine-specific responses) (www.esid.org) with no other secondary cause identified. Secondary causes are reasonably excluded where there is no evidence of monoclonality (normal serum electrophoresis, urine Bence Jones Protein negative), haematological malignancy such as chronic lymphocytic leukaemia (normal FBC), and no evidence of protein loss from the gut or kidney (normal serum albumin and total protein, absence of significant proteinuria or evidence of intestinal malabsorption). 
TABLE I Clinical presentations. The absence or dysfunction of different compartments of the immune system results in typical clinical presentations, which can be used to direct investigation.

\begin{tabular}{|l|l|l|l|}
\hline Presentation & Possible diagnosis & Investigations & Specialist investigations \\
\hline $\begin{array}{l}\text { Encapsulated bacteria (oto- } \\
\text { sino-pulmonary infection) }\end{array}$ & $\begin{array}{l}\text { Humoral deficiency } \\
\text { Rarely innate immuno- } \\
\text { deficiency }\end{array}$ & $\begin{array}{l}\text { Total Ig G,A, M } \\
\text { FBC (haematological malignancy or } \\
\text { autoimmune cytopenias) } \\
\text { SEP/BJP (exclude myeloma) } \\
\text { Albumin, total protein, urinary protein } \\
\text { (exclude protein-losing state) }\end{array}$ & $\begin{array}{l}\text { Specific responses } \\
\text { lgG subclasses } \\
\text { Lymphocyte subsets } \\
\text { Extended B cell markers }\end{array}$ \\
\hline $\begin{array}{l}\text { Opportunistic infection } \\
\text { (e.g. severe warts, candidiasis, } \\
\text { pneumocystis) }\end{array}$ & $\begin{array}{l}\text { T cell deficiency } \\
\text { Rarely innate immuno- } \\
\text { deficiency }\end{array}$ & As above plus lymphocyte subsets & $\begin{array}{l}\text { Analysis for known } \\
\text { mutations causing SCID }\end{array}$ \\
\hline $\begin{array}{l}\text { Recurrent invasive } \\
\text { Staphylococcus or aspergillus }\end{array}$ & $\begin{array}{l}\text { Phagocyte deficiency } \\
\text { Rarely innate immuno- } \\
\text { deficiency }\end{array}$ & As above & $\begin{array}{l}\text { Neutrophil respiratory } \\
\text { burst assay } \\
\text { Cytokine analysis }\end{array}$ \\
\hline Atypical mycobacteria & $\begin{array}{l}\text { T cell deficiency } \\
\text { MSMD } \\
\text { Rarely innate immuno- } \\
\text { deficiency }\end{array}$ & As above & $\begin{array}{l}\text { Cytokine analysis testing } \\
\text { ILI2/interferon gamma } \\
\text { pathways }\end{array}$ \\
\hline
\end{tabular}

Abbreviations: BJP: Bence Jones protein; FBC: full blood count; lg: immunoglobulin; MSMD: Mendelian susceptibility to mycobacterial diseases; SCID: severe combined immune deficiency; SEP: serum electrophoresis.

Common variable immunodeficiency is the commonest PID and is diagnosed when the clinical presentation of recurrent bacterial infection occurs with: (I) low IgG, together with low IgA and/or IgM; (2) poor vaccine responses; and (3) no secondary cause identified. ${ }^{8}$ It is a heterogeneous condition of unknown cause, likely to represent a number of single or polygenic mutations. The clinical features and disease evolution are highly variable. Approximately 10\% of patients have an affected family member, but a causal mutation has been demonstrated in only a few families. ${ }^{9}$ Of the patients meeting the criteria for CVID, 10-30\% will have evidence of a combined immune deficiency or immune dysregulation. This may present clinically with recurrent fungal or viral infections, inflammatory (GLILD) or autoimmune conditions or lymphoproliferative disease, or may just become apparent when functional or quantitative lymphocyte analyses are performed. ${ }^{10}$

The diagnosis of CVID automatically qualifies the patient for immunoglobulin replacement, which has been unequivocally shown to reduce infections, improve life quality and prolong life expectancy. ${ }^{5,10}$ In individuals with severe or frequent recurrent infections but normal levels of total immunoglobulin, it may also be warranted to check IgG subclasses and to investigate antibody responses to vaccination; either subclass deficiency or specific antibody deficiency may, in the correct clinical context, qualify patients for a trial of antibody replacement. If such trials have a measurable positive effect on predetermined clinical outcomes (such infection frequency, antibiotic use, hospital admissions) they may be extended to the long term.
When to measure specific antibody levels

Reduced vaccine responses are a common finding in PAD, and they are a useful measure of the severity of humoral deficiency in cases where the total IgG is normal or only marginally reduced. These measurements are required for the diagnosis of CVID, and may also be indicated in the context of recurrent infections with normal total immunoglobulin infections, or in the setting of bronchiectasis. Testing may not be available in all centres and may necessitate referral to specialist units. Most immunologists quantify specific functional antibodies before, and four to eight weeks following, protein or polysaccharide-protein conjugate vaccines (tetanus, Haemophilus, tested with vaccines such as Revaxis, Menitorix) and polysaccharide vaccines (Pneumovax).

It is important to note that vaccination against Haemophilus influenzae Type B offers no clinical protection against respiratory infections, which are due to non-typeable strains of this organism. The presence of immunity to exanthematous viral illnesses (e.g. Varicella zoster virus, VZV) may also be assessed. This profile tests the $B$ cell, $T$ cell and memory elements of humoral immunity. Symptomatic patients with reduced responses to vaccines and normal total IgG are diagnosed with a specific antibody deficiency and may be candidates for a trial of antibody replacement.

Selective $\lg A$ deficiency, selective $\lg M$ deficiency and IgG subclass deficiencies may also be identified in patients with recurrent respiratory tract infection, although they can also be seen in entirely healthy individuals. IgA deficiency is in fact present in one in 300 of the general UK population, and in the vast majority has no clinical sequelae. However, once again depending on infection frequency and severity, an immunologist may decide to 
undertake a trial of immunoglobulin replacement for these conditions, with defined criteria to assess the clinical response.

\section{Management of PAD}

Immunoglobulin replacement therapy and prompt recognition of infection are the mainstays of treatment. Immunoglobulin replacement must be approved, instituted and supervised by a clinical immunologist, and can be undertaken by the intravenous (usually every three to four weeks) or subcutaneous (usually weekly or fortnightly) route. Infections should be investigated where possible to identify the causative organism, but this should never delay the institution of prompt and effective treatments (in all but exceptional circumstances, patients should have immediate access to a 'reserve' course of high dose antibiotics for 10-14 days, the particular agent selected on the basis of previous bacterial isolates where possible or previous response patterns where not). The aim of such intervention is to reduce the frequency and severity of breakthrough infections and thus to minimise the onset or progression of structural lung disease.

A proportion of patients will need prophylactic antibiotics to prevent breakthrough infection despite replacement immunoglobulin at optimised doses. This is more common in the presence of structural lung disease and is guided by bacterial isolates, local protocols, patient tolerability and efficacy; common regimens include doxycycline $100 \mathrm{mg}$ od, amoxicillin $250-500 \mathrm{mg}$ bd, clarithromycin 250-500 mg bd, azithromycin $250 \mathrm{mg}$ thrice weekly. Treatment should be tailored to patient response and adjusted where clinical circumstances demand.

High-resolution computed tomography (HRCT) is normally undertaken before or shortly after diagnosis with PAD and recurrent infections, to establish the presence or absence, severity and distribution of bronchiectasis. The frequency with which HRCT should be undertaken for monitoring has not been established, but most specialists would perform assessment more frequently in patients who are declining symptomatically or experiencing recurrent infections, despite the measures outlined above. Radiological screening will also document whether GLILD is present and may reveal the presence of lymphoproliferative disease. Serial lung function testing may also be helpful in the setting of progressive or symptomatic lung disease."

Immunoglobulin replacement therapy does not treat or prevent the occurrence or complications of immune dysregulation (GLILD, nodular regenerative hyperplasia of the liver, autoimmune disease). Specialists with relevant expertise should treat these conditions on an individual basis.

\section{T CELL DEFICIENCY}

\section{When to suspect $T$ cell deficiency}

Clinical presentation varies, depending on both the underlying immune component affected and the severity of the defect. Opportunistic infection without a known underlying cause should prompt $\mathrm{T}$ cell analysis and referral for specialist opinion.The pattern of opportunistic infection in patients with advanced HIV resembles that seen in patients with non-HIV T cell deficiency. Milder T cell deficiencies present with recurrent warts (due to human papilloma virus infection), mucocutaneous candidiasis, and intracellular organisms normally cleared by $\mathrm{T}$ cell-activated-macrophages (e.g. non-tuberculous Mycobacteria spp or Salmonella spp). More profound deficits may present with less virulent organisms (atypical mycobacteria, Cryptococcus neoformans, Toxoplasma gondii, Pneumocystis jirovecii) or severe illness - candidaemia, aspergillosis, and systemic or severe infection with members of the herpes virus family. ${ }^{12}$

\section{How to investigate $T$ cell deficiency}

Human immunodeficiency virus lymphocyte subsets generally enumerate total lymphocytes (CD45), $T$ cells (CD3), $T$ helper (CD4), cytotoxic T cells (CD8) and B cells (CDI9), and most laboratories include NK cells (CDI6/56) in the count. Further analysis will include functional $T$ cell assessment (proliferation and receptor diversity) - these assays will usually only be possible in specialist centres. Relevant genetic screening for known mutations can be guided by the pattern/severity of lymphopenia. Significant $T$ cell deficiency is often accompanied by impaired humoral function or immune dysregulation, and hence tests for $B$ cell function should also be undertaken as documented above. ${ }^{12}$

\section{Management of $T$ cell deficiency}

Patients with profound lymphopenia should receive prophylaxis as informed by HIV epidemiology, for example PCP prophylaxis should be considered for CD4 counts $<200$ and CMV prophylaxis for CD4 counts $<50$. Secondary anti-fungal prophylaxis may also be required. There are no national guidelines for PID-associated $T$ cell lymphopaenia, and local protocols may offer more detailed management advice.

New onset symptoms of infection should be taken very seriously in a patient with a known T cell deficiency and the infection screen should be as broad and thorough as in patients with HIV or haematological malignancy.These patients may not present with classic symptoms of infection or inflammation, may be diagnosed with multiple pathogens simultaneously, and can succumb rapidly to overwhelming infection if prompt and effective treatment is not initiated. 


\section{DEFECTS OF INNATE IMMUNITY}

These include abnormalities in the complement cascade, phagocytic function and Toll-like receptor signalling. The presentation varies depending on which element is impaired; there are many rare conditions in this group and for the purposes of this review we will limit discussion to those syndromes most likely to present through the recurrent respiratory or other infection. As a general rule, the combination of severe or recurrent staphylococcal and/or fungal infection of the lung and skin should raise the suspicion of a phagocyte defect or hyperimmunoglobulin E syndrome (HIES), particularly in the context of a relevant family history.

\section{PHAGOCYTE DEFICIENCIES}

These are rare conditions, but the consequences of a missed diagnosis can be severe. Presentation ranges from mucocutaneous candidiasis to recurrent severe invasive infections with Staphylococcus spp or Aspergillus spp. Phagocytes generate a rapid response to infection and initiate local and systemic inflammation; hence as well as being uniquely susceptible, these patients often lack the classical symptoms and signs of bacterial invasion. They may feel relatively well, with a low CRP and white cell count, despite extensive bacterial load.

Chronic granulomatous disease (CGD) patients suffer recurrent pneumonia, abscesses, osteomyelitis, adenitis and skin infections. ${ }^{13}$ About $70 \%$ of CGD results from an $X$-linked condition, and the majority of patients present in childhood, but autosomal recessive inheritance also occurs and milder phenotypes can present late into adulthood. This condition affects the ability of phagocytic cells to generate reactive oxygen species, resulting in reduced pathogen killing. As a result, these patients are unable to clear Staphylococcus aureus and Aspergillus spp in particular, as well as other intracellular organisms (including Nocardia spp, Salmonella spp and Mycobacteria spp). Granuloma formation and autoimmunity are also common and there may be a family history of systemic lupus erythematosus in female carriers who do not have the disease itself.

Diagnosis is based on the finding of an abnormal neutrophil respiratory burst (Phagocye Oxidase test available in specialist centres). The definitive treatments for childhood presentation are bone marrow transplantation or gene therapy. For those presenting as adults, the principles of antimicrobial prophylaxis (usually with co-trimoxazole or co-amoxiclav plus itraconazole), early diagnosis of infections and aggressive management of infectious complications are the mainstay of management, although transplant may be considered in exceptional circumstances. Interferon-gamma is licensed to treat severe fungal infections in CGD, but unlike in the USA, the European consensus is that it should not be used for routine prophylaxis.
Hyperimmunoglobulin E syndrome (formerly known as Job's syndrome) predisposes to a broad range of Grampositive and Gram-negative infections, most commonly of the skin, joints and respiratory tract. ${ }^{14}$ In the chest, staphylococcal pneumonia complicated by pneumatocoele formation or bronchiectasis is the classic manifestation. Secondary colonisation or invasive infection of pneumatocoeles/damaged lung with bacteria such as Pseudomonas aeruginosa and fungi such as Aspergillus fumigatus may lead to challenging long-term management problems. Patients with HIES may also develop a characteristic skin rash at birth, experience delayed or absent shedding of their primary dentition and suffer life-long severe eczema; they often have characteristic 'leonine' facies, with a protuberant forehead, broad nose and prognathia.

Patients with suspected HIES should be referred for specialist assessment. The diagnosis is based on clinical findings and confirmed by genetic testing; inheritance may be autosomal dominant (STAT3 mutations) or recessive (DOCK8 mutations). Management should be supervised by a clinical immunologist and usually combines prophylactic antibiotics plus anti-fungals, prompt treatment of acute infections, surgical management of structural lung disease where necessary. Input from a respiratory specialist is often required. ${ }^{14}$

\section{COMBINED IMMUNODEFICIENCY DISORDERS}

Adult onset immune deficiency states often present with symptoms suggesting combined defects involving both $B$ and $T$ cells, which may be confirmed using the laboratory investigations outlined above. A proportion of these will be late presentations of known conditions that more usually present as a severe combined immune deficiency (SCID) in childhood. More commonly, a previously healthy adult will present with progressive symptoms (e.g. recurrent respiratory tract infections) that prompt the initial immune screen, and identify a combination of underlying abnormalities (e.g. reduced IgG levels and CD4 lymphopenia).

Fulminant presentations do occur, and the lack of a previous history of infection does not preclude the diagnosis, although it is clearly critical to exclude secondary immune deficiency and, in particular, HIV infection in this setting. Involvement of a clinical immunologist in such cases can expedite diagnosis and the institution of specific (and potentially life-saving) treatments.

\section{ACTIVATED PI3 KINASE DELTA SYNDROME}

\section{Discovery}

Since 2005, the availability of next-generation DNAsequencing platforms has made it possible to determine in a cost-effective and timely fashion nearly all of the 
coding variation present in an individual human genome, a process termed 'exome sequencing'. A large number of genetic mutations have been found using such techniques, but many are restricted to one or two families or to specific racial or ethnic groups.

Using whole exome sequencing to screen patients with severe familial PID but no known genetic mutation, we recently found that patients from two unrelated families (from England and Scotland) shared the same activating mutation in the gene coding for the catalytic plIOS subunit of phosphoinositide 3-hydroxykinase delta

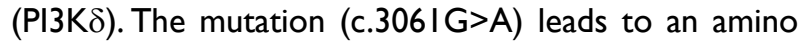
acid substation in the protein (glutamic acid for lysine at position I02I) and is hence termed EI02IK.

With further screening, we identified the El02IK mutation in I7 PID patients from five unrelated European families from different racial backgrounds. One patient had healthy biological parents (confirmed using genomewide identity-by-descent analysis) who did not carry the mutation, hence in this case the mutation must have arisen de novo. We have termed this syndrome the Activated PI3 Kinase Delta Syndrome (APDS).

Following the publication of our findings, ${ }^{15}$ two groups from the USA have identified three additional activating mutations; ${ }^{16,17}$ the 17 patients reported in these publications were ethnically diverse. To date, a total of 35 patients from a diverse range of ethnic and racial backgrounds have now been reported to suffer from APDS; further cases are likely to be diagnosed as awareness and testing are broadened.

\section{Clinical features}

Patients with APDS have a phenotype that is suggestive of a combined $B$ and $T$ cell disorder. ${ }^{15-17}$ The most consistent reported clinical feature is recurrent otosino-pulmonary infection with Haemophilus influenzae and Streptococcus pneumoniae (characteristic of B cell dysfunction). Resulting end organ damage is common, including bronchiectasis and hearing impairment. A major subset of these patients has experience severe, even life-threatening infections with herpes-family viruses, including persistent CMV or EBV viraemia, EBV colitis, HSV pneumonitis and severe VZV; other patients have developed recurrent warts (human papilloma virus) or molluscum contagiosum (mollluscum contagiosum virus). These viral infections are typical of a defective $T$ cell- and perhaps NK cell-mediated immunity. Other reported manifestations have included cellulitis and minor skin abscesses, but no serious fungal infections; so a phagocyte defect, if present, is subtle.

In addition to immune deficiency, these patients exhibit features of immune dysregulation, with frequent splenomegaly and lymphadenopathy (and, in some cases, dramatic mucosal lymphoid hyperplasia). Immune cytopaenias are seen in about $10 \%$ of the reported patients, and one patient developed an immune complex glomerulonephritis resulting in end-stage renal failure. Importantly, there is a high incidence of lymphoproliferative disease (nine lymphomas from 35 patients with APDS), with both EBV-driven and EBV-negative lymphomas described.

\section{Immunological features}

Immunologically, these patients have elevated levels of IgM (indeed, several patients had previously been diagnosed with the Hyper IgM syndrome, a combined immunodeficiency disorder in which there is a failure of class switch recombination leading to elevated IgM but decreased $\lg G$, IgA, plus evidence of $T$ cell dysfunction). They exhibit defective or absent antibody responses to polysaccharide vaccines. IgG levels are usually in the normal range, but in cases where IgG subclasses have been measured, an IgG2 subclass deficiency almost universal. Some, but not all patients display reduced IgA levels. Cellular phenotyping demonstrates a reduction in circulating class switched memory $B$ cells with an increase in immature transitional B cells, and the circulating $T$ cells display a senescent phenotype. In vitro, patient-derived T cells exhibit impaired proliferation responses.

\section{Biochemical and cellular features}

Biochemically, the fact that these mutations act to increase PI3K activity has been confirmed by in vitro methods and by measuring elevated PIP ${ }^{3}$ levels and increased PIP $^{3}$-dependent downstream signalling (including the AKT- and mTOR pathways) in patientderived lymphocytes. Patient $T$ cells exhibited defective cytokine production, and both $\mathrm{B}$ and T-lymphocytes exhibited a survival defect in vitro. The impact of the pl IOS mutations on antibody class switching (from low affinity $\lg M$ to high affinity $\operatorname{lgG} / \mathrm{A}$ ) is unclear, with one group reporting this response to be impaired and another reporting it to be preserved; these inconsistencies may reflect the examination of patients with different genotypes or methodological differences. Importantly, isoform-selective PI3K $\delta$ inhibitors could reverse many of the biochemical and functional abnormalities seen in the patient cells.

\section{Potential treatment options}

Many of these patients have experienced recurrent and debilitating infections, and in many cases there is a strong family history of early death from infection-related causes. In several cases, the patients have continued to suffer infections and progressive end-organ damage despite intensive treatment with strategies including antibiotic and antiviral prophylaxis, intermittent or regular intravenous antibiotics, and immunoglobulin replacement.

One patient who underwent a bone marrow transplant was reported to be well 18 months following the procedure. However, not all patients may be suitable for such intervention, particularly adults or those with a 
milder phenotype. One patient treated with rapamycin ${ }^{16}$ was reported to show regression of lymphadenopathy and hepatosplenomegaly, associated with an improved circulating $T$ cell profile. While the side effect profile of rapamycin may preclude its longer-term use in this infection-prone group, intense pharmaceutical industry interest in the therapeutic potential of isoform-selective PI3K $\delta$ inhibitors for malignant and inflammatory disease has recently culminated in the publication of the first clinical trials of these compounds in hematological malignancy, ${ }^{18,19}$ and other compounds are in development.

In future we hope that a targeted therapy with an acceptable side effect profile will be available to these patients in the longer term. Furthermore, the significance of these findings to other patient groups, such as those with recurrent respiratory infection in the presence or absence of structural lung disease, remains to be explored.

\section{SUMMARY}

Patients with PIDs may present in adult life, and the alert clinician can recognise the clinical patterns that may suggest this diagnosis. Particular triggers to arouse suspicion include recurrent oto-sino-pulmonary infection (seen in disorders of B cell antibody-mediated

\section{REFERENCES}

I Edgar JD, Buckland M, Guzman D et al. The United Kingdom Primary Immune Deficiency (UKPID) Registry: report of the first 4 years' activity 2008-20I2. Clin Exp Immunol 20I4; 175:68-78. http://dx.doi.org/I0.IIII/cei.I2I72

2 Holland SM. The cup runneth over: lessons from the everexpanding pool of primary immunodeficiency disease. Nat Rev Immunol 2013; I3:635-48. http://dx.doi.org// 0.1038/nri3493

3 Shearer WT, Dunn E, Notarangelo LD et al. Establishing diagnostic criteria for severe combined immunodeficiency disease (SCID), leaky SCID, and Omenn syndrome:The Primary Immune Deficiency Treatment Consortium experience. J Allergy Clin Immunol 2014; 133:1092-8. http://dx.doi.org//0.1016/j.jaci.2013.09.044

4 Jolles $S$. The variable in common variable immunodeficiency: a disease of complex phenotypes. J Allergy Clin Immunol Pract 2013; I:545-56. http://dx.doi.org/I0.1016/j.jaip.2013.09.0I5

5 Busse PJ, Razvi S, Cunningham-Rundles C. Efficacy of intravenous immunoglobulin in the prevention of pneumonia in patients with common variable immunodeficiency. J Allergy Clin Immunol 2002; 109:1001-4.

6 Ballow M. Primary immunodeficiency disorders: antibody deficiency. J Allergy Clin Immunol 2002; 109:58I-9I.

7 Webster AD. Clinical and immunological spectrum of common variable immunodeficiency (CVID). Iran J Allergy Asthma Immunol 2004; 3:103-13.

8 Conley ME, Notarangelo LD, Etzioni A. Diagnostic criteria for primary immunodeficiencies. Representing PAGID (Pan-American Group for Immunodeficiency) and ESID (European Society for Immunodeficiencies). Clin Immunol 1999; 93:190-7.

9 Park JH, Resnick ES, Cunningham-Rundles C. Perspectives on common variable immune deficiency. Ann N Y Acad Sci 2011; | 246:4 I-9. http://dx.doi.org/ I0. I I I I/j. I749-6632.20 I I.06338.x

I0 Resnick ES, Moshier EL, Godbold JH et al. Morbidity and mortality in common variable immune deficiency over 4 decades. Blood 2012 I 19:1650-7. http://dx.doi.org/I0. I | 82/blood-20 I I-09-377945 immunity), opportunistic infection (characteristic of $T$ cell defects) and recurrent cutaneous and pulmonary infection with staphylococcus and/or fungal pathogens (phagocyte disorders/HIES). Excluding causes of secondary immune deficiency and undertaking simple investigations such as circulating immunoglobulin levels, lymphocyte subsets and a full blood count, will exclude the majority of severe PIDs.

Patients with abnormal investigations or with a suggestive clinical phenotype should be discussed with, or referred to, a clinical immunology service for further investigations and for the initiation of treatment and monitoring where appropriate. Immunoglobulin replacement, targeted prophylaxis and prompt, aggressive management of infections have transformed the life quality and life expectancy of adult PID patients.

Finally, new insights into the pathogenesis of PIDs are being identified by next-generation DNA sequencing techniques; as an example, a severe combined immunodeficiency due to an activating mutation in PI3K $\delta$ has recently been identified in a relatively high proportion of PID patients screened, and this condition may be amenable to specific pharmacological intervention.

I I Park JH, Levinson Al. Granulomatous-lymphocytic interstitial lung disease (GLILD) in common variable immunodeficiency (CVID). Clin Immunol 2010; I34:97-103. http://dx.doi.org/I0.1016/j. clim.2009.10.002

12 Bousfiha AA, Jeddane L, Ailal F et al. A phenotypic approach for IUIS PID classification and diagnosis: guidelines for clinicians at the bedside. J Clin Immunol 2013; 33:1078-87. http://dx.doi. org/I0.1007/s I0875-013-990I-6

13 Holland SM. Chronic granulomatous disease. Clin Rev Allergy Immunol 2010; 38:3-I0. http://dx.doi.org/I0.I007/s/2016-0098136-z

14 Yong PF, Freeman AF, Engelhardt KR et al. An update on the hyperIgE syndromes. Arthritis Res Ther 2012; 14:228.

15 Angulo I, Vadas O, Garçon F et al. Phosphoinositide 3-kinase $\delta$ gene mutation predisposes to respiratory infection and airway damage. Science 2013; 342:866-7I. http://dx.doi.org/I0.II26/ science. 1243292

16 Lucas CL, Kuehn HS, Zhao F et al. Dominant-activating germline mutations in the gene encoding the $\mathrm{PI}(3) \mathrm{K}$ catalytic subunit pI I0 result in $\mathrm{T}$ cell senescence and human immunodeficiency. Nat Immunol 2014; 15:88-97. http://dx.doi.org//0.1038/ni.277|

17 Crank MC, Grossman JK, Moir S et al. Mutations in PIK3CD can cause hyper IgM syndrome (HIGM) associated with increased cancer susceptibility. J Clin Immunol 20I4; 34:272-6. http://dx.doi. org/I0.1007/s I0875-0I4-00I2-9

18 Gopal AK, Kahl BS, de Vos S et al. PI3K $\delta$ inhibition by idelalisib in patients with relapsed indolent lymphoma. N Engl J Med 2014; 370: 1008-18. http://dx.doi.org/ I0.1056/NEJMoa I 3 | 4583

19 Furman RR, Sharman JP, Coutre SE et al. Idelalisib and rituximab in relapsed chronic lymphocytic leukemia. N Engl J Med 2014; 370:997-1007. http://dx.doi.org/I0.I056/NEJMoa I 3 I 5226 